\title{
LOS ESTUDIOS SEMIÓTICOS EN MÉXICO
}

\author{
Adrián S. Gimate-Welsh 1 \\ Universidad Autónoma Metropolitana, Iztapalapa (México) \\ Para la pareja Suárez-Lastra, maestros incansables \\ de los estudios lingüísticos y sociolingúísticos en México
}

\section{INTRODUCCIÓN}

El itinerario semiótico del México contemporáneo puede caracterizarse como una fase de expansión y consolidación del esfuerzo individual y colectivo de mujeres y hombres de diversas orientaciones teóricas que los une y reafirma en unos casos y los distingue en otros.

Llegar a esta etapa no ha sido fácil, como tampoco lo ha sido nuestro origen - lleno de partos difíciles, desarrollos desiguales y asechanzas múltiples - pues se han tenido que romper inmovilismos en distintos ámbitos disciplinarios e interdisciplinarios y se han tenido que derribar concepciones feudales en múltiples casos institucionales. Hemos llegado a este momento, con nuestras cruces en la espalda, pero con una mística que está por encima de lo material; mística que

1 Presidente de la Asociación Mexicana de Estudios Semióticos. Investigador Nacional, Nivel III. 
seguramente fue el alimento de San Cristóbal de las Casas, de Palafox y Mendoza o de Junípero Serra.

Los pasos de nuestros antepasados - los pobladores de la Nueva España-como Alonso de la Vera Cruz, primer semiotista americano, y los practicantes de la retórica sacra ${ }^{2}$, constituyen los primeros paradigmas de la semiótica. Más aun, por su composición pluriétnica, podríamos decir que el México contemporáneo es un laboratorio semiótico en el que se perciben cruces de sistemas semióticos distintos.

De los antecedentes recientes sobre la semiótica en México, cabe mencionar el ensayo «Semiotics in México» ${ }^{3}$, de Regina JiménezOttalengo en el que señala que «semiotics has been practiced in Mexico since about 1976», relacionando esta práctica semiótica con los trabajos del seminario de semiótica dirigido por Renato Prada Oropeza en la Universidad Veracruzana, el Instituto de Investigaciones Filológicas de la Universidad nacional Autónoma de México y el área de comunicación de la Universidad Autónoma MetropolitanaXochimilco y los estudios sociolinguiísticos coordinados por Oscar Uribe Villegas, del que formaba parte Jiménez-Ottalengo. Podemos apreciar, como veremos más adelante, tres líneas de investigación y docencia: los estudios literarios que tiene como referentes a los teóricos del estructuralismo de los años sesenta: Barthes, Todorov,

2 Para una muestra de los libros que circulaban en la colonia mexicana mencionemos los siguientes: de Josepho Mariano Vallarta, De arte rhetorica y poetica, Qui in Regali \& Antiquiori Divi Ildefonsi Collegio Mexicano Literarum Studijs operan navant, Mexici, 1753; Ventjas de la elocuencia popular, del italiano Juan Antonio Muratori, traducida al español por Vicente María de Tercilla, Joachim Ibarra, s/a; de Juan Petreio, Progymnasmata artis rhetoricae, 1539, s.imp., s.l.; de Francisco Antonio Pomey, Candidatus rhetoricae, Venezia, 1734; de Petro-Maria La-Torre, retórica y poesía, 1753; de Juan Díaz Rengifo, Arte poética española, 1803, impresor Ma. Martí Vda., Barcelona; de Pablo Antonio González y Fabro, Divertimento rhetorico ciceroniano, o erudita diadema oratoria, 1752, Juan de Zúñiga, Madrid; Joachim Díaz Betancourt, Luz de predicadores, 1752, Viuda de don Joseph Bernardo, de Hogal, México; Fray Fco. De la Concepción Barbosa, Manual de predicadores, 1728, Herederos de la Vda. De Miguel Rivera, México; Mariano Madramany, Tratado de la elocución o del perfecto lenguaje y buen estilo respecto del castellano, 1795, Hermanos de Orga, Valencia, y Compendio de la rhetorica en que se da un fácil, y utilisimo methodo de enseñar el arte oratoria, 1776, Barcelona, traducción de Fr. Raymundo Josef Rebollida; Josef Gómez Hermosilla, Arte de hablar en prosa y en verso, 1869, Libería de Rosa y Bouret, París; Antonio Gil Zárate, Manual de literatura. Principios generales de poética y retórica, 1865, Librería de Garnier Hermanos, París, 7a. edición corregida y aumentada. Su primera edición es de 1844, Boix, Madrid. Estos son pues sólo algunos de los ejemplos de textos coloniales que forman parte de los fondos reservados de la Universidad Nacional Autónoma de México, de la Benemérita Universidad Autónoma de Puebla y de la Universidad Autónoma de Oaxaca.

3 Cf. The Semiotic Sphere, edited by Thomas Sebeok y Jean Umiker-Sebeok, New

York, London: Plenum Press, 1986, pp. 359-368. 
Bremond, Greimas, Segre y a los formalistas rusos, los estudios inscritos en la temática del lenguaje y la sociedad y los estudios semióticos en su relación con la retórica clásica, neorretórica y el estructuralismo según se desprende de los trabajos publicados por los diversos investigadores. Aun cuando el Centro de Ciencias del Lenguaje del Instituto de Ciencias de la Universidad Autónoma de Puebla ya estaba en funciones, así como la Maestría en Ciencias del Lenguaje se encontraba operando y se habían publicado varios números de la Colección Signo y Sociedad, este ensayo no registra su existencia ${ }^{4}$.

En The Semiotic Web 1987, editado por Thomas Sebeok y Jean Umiker-Sebeok ${ }^{5}$, Beatriz Garza Cuarón en su artículo «Semiotics in Mexico» (Garza Cuarón, 1988: 267-305), hace referencia a la pluralidad semiótica de México basada en la pervivencia de los grupos étnicos del periodo de la conquista hasta nuestros días. La vitalidad de estas lenguas se constata en el número de lenguas indígenas que aún se hablan a lo largo y ancho del país: 201 en total y la existencia de 56 etnias, algunas de las cuales hoy demandan su autonomía cultural y lingüística. En este contexto, resulta lógico que los colonizadores españoles tuvieron que recurrir a la redacción de manuales de gramática y diccionarios a fin de llevar a cabo la conquista y la cristianización de los nuevos súbditos de la Corona Española. Esto implicó la descripción de sistemas simbólicos múltiples. Vemos pues la importancia de los manuales de retórica, de poética y por supuesto la gramática. El paradigma de estas obras lo constituyó, como bien sabemos, el pensamiento de Nebrija quien exhibe una conciencia sociolingüística en sus finalidades de enseñar latín o castellano, a saber:

Yo hago lo siguiente: me pongo en el caso de aquéllos a quienes quiero enseñar, y no digo ni escribo nada que los niños no puedan entender sin omitir tampoco nada que sea necesario para iniciarlos en la lengua latina ${ }^{6}$.

Idea que reafirma cuando se dirige a la reina Isabel de Castilla:

Para que después que vuestra alteza metiere debajo de su yugo muchos pueblos bárbaros y naciones de peregrinas lenguas, y con el vencimiento aquéllos tenían necesidad de recibir las leyes que el vencedor pone al vencido,

${ }^{4}$ En 1985, conjuntamente con la Facultad de Filosofía y Letras de la UNAM, el Centro de Ciencias del Lenguaje y la Facultad de Filosofía y Letras organizan el I Congreso Internacional Latinoamericano de Semiótica en el que participan, entre otros estudiosos, Paolo Fabbri, Eric Landowski, Mario Valdés, etc.

5 Berlín, Amsterdam, New York: Mouton de Gruyter, 1988.

6 Cf. Introductione latinae, 1481. 
y con ellas nuestra lengua, entonces por este arte podrán venir en el conocimiento de ella como ahora nosotros dependemos el arte de la gramática latina para deprender el latín ${ }^{7}$.

Está claro que la idea reinante de su tiempo subrayaba la conquista lingüística de los pueblos sometidos por la espada. Como bien sabemos, su tarea fue más fácil al reconocer que la mayoría de los pueblos indios habían estado sometidos a la lengua de los aztecas —l náhuatl-. De ahí que utilizaran el náhuatl en sus propósitos administrativos y religiosos. Es decir, la imposición de la lengua española fue lenta y sutil (Aguirre Beltrán, 1983: 37-44). Así pues, no obstante la orden de Carlos I en 1550 de cristianizar a los indios por medio de la lengua española o el latín, los misioneros continuaron su evangelización en las lenguas vernáculas (Garza Cuarón, 1988: 270). Así los franciscanos, que llegaron a la colonia en 1524, para 1570 habían escrito más de 80 libros relacionados con la gramática, el léxico y traducciones de la Biblia, tendencia que continuó en los siglos XVII y XVIII.

Son muchos los estudios sobre lingüística de las lenguas indígenas en el siglo XX, pero resaltemos sólo los que llevó a cabo el gran teórico del relativismo lingüístico, Benjamin Lee Whorf (1897-1941). Así pues, en el contexto del XXII Congreso Internacional de Americanistas, en 1928, lee los trabajos «An Aztec account of the period of the toltec decline»y «Aztec linguistics.» Su interés se extendió al estudio de los jeroglíficos del maya y del náhuatl. Tales estudios lo llevaron a concluir que hay semejanzas entre los jeroglíficos del calendario de los aztecas y los de la cultura maya. Escribió otros artículos sobre el tema, pero destaca el ensayo «Decipherment of the linguistic portion of the Maya hieroglyphs», publicado en 1940.

Desde una reflexión sobre los signos, en el marco de la comunicación, Tzvetan Todorov concibe el descubrimiento de América como un mirarse a sí mismo, como un descubrimiento del uno en el otro ${ }^{8}$.

Sin duda alguna, una de las instituciones pioneras en los estudios semióticos principios del siglo XX corresponde al Instituto Nacional de Antropología e Historia que tiene como antecedente el International

7 Cf. G. Félix Maldonado, Elio Antonio de Nebrija. Debelador de la barbarie, comentador eclesiástico, pedagogo y poeta, Madrid, 1942, p. 24.

8 La conquista de América. La cuestión del otro, traducción de Flora Botton Burlá, México: Siglo XXI, 1987. 
School of American Archeology and Ethnology 9 . Dicha tradición fue continuada por el destacado lingüista Mauricio Swadesh -discípulo de E. Sapir-, teórico de la glotocronología. Su presencia en México está ligada además a la introducción del estructuralismo. Una segunda línea de estudios semióticos está vinculada a la presencia en México del Instituto Lingüístico de Verano en $1935^{10}$. Sus trabajos semióticos, que tenían una finalidad religiosa, son fundamentales para el conocimiento de las lenguas indígenas de México. De las figuras más sobresalientes cabe mencionar al linguiista norteamericano Kenneth Pike, teórico fundamental de la Tagmémica. Como muestra de sus aportes mencionemos Language in Relation to a Unified Theory of the Structure of Human Behavior (1967) y a sus discípulos J. Grimes, R. Langacre y V. Pickett. De esta última sobresale su Manual of Morphology, herramienta de análisis tagmémico de lenguas indígenas. $\mathrm{El}$ interés por los estudios de las lenguas amerindias lo encontramos asimismo en Jorge Suárez, sobre todo en el ámbito de la geolinguíística indígena y Yolanda Lastra en la dirección de la sociolingüística ${ }^{11}$. Su obra se inscribe - habrá que subrayarlo- en una amplia experiencia en la investigación y la docencia en los estudios lingüísticos y antropológicos y se instala en pleno desarrollo del estructuralismo en las instituciones de educación superior en México. Directa o indirectamente, muchos de los lingüistas y semiotistas mexicanos somos discípulos de la pareja Suárez-Lastra.

\section{LA ERA DEL ESTRUCTURALISMO}

De acuerdo con Garza Cuarón, el estructuralismo se inicia en México con la llegada de Raimundo Lida (1947) - traductor del Curso de Lingüística General de F. de Saussure-y su presencia en El Colegio de México. El impulso estructuralista fue más notorio sin embargo a partir de la década de los sesenta con la presencia de B. Pottier (1966),

\footnotetext{
9 Fundado en 1910. El Director de ambas instituciones fue el eminente linguiista Franz Boas.

${ }_{10}$ William Cameron Townsend fue su fundador.

11 Cf. Sociolingüística para hispanoamericanos. Una introducción, México: El Colegio de México, 1992. Si bien todos los capítulos del libro son importantes, para nuestro interés resulta interesante el capítulo «La lengua como medio de comunicación» en el que se tratan temas como «Etnicidad e identidad», «Etnografía de la comunicación», «Interacción social».
} 
el rumano Eugenio Coseriu $(1967,1973)$, los alemanes Klaus Heger (1968) y Kurt Baldinger (1972). En este contexto se propagó el estudio lexicológico, semántico, filológico y dialectológico con la presencia de los españoles Juan M. Lope Blanch, Manuel Alvar y Gregorio Salvador.

En la década de los setenta, en instituciones como la Universidad Nacional Autónoma de México, la Universidad Autónoma de Puebla, la Universidad Autónoma Metropolitana-Xochimilco y la Universidad Veracruzana, se integran grupos de investigación y docencia en los campos de los estudios literarios y semióticos, pero con una fuerte influencia estructuralista. Los objetos de estudios son el texto literario, el texto político, el texto mediático y temáticas relacionadas con la antropología y la sociología.

\section{LOS ESTUDIOS LITERARIOS}

1978 es un año clave para el impulso de los estudios semióticos en México. José Pascual Buxó funda el Seminario de Poética en la UNAM. Sus miembros, además de su director y fundador, fueron: Helena Beristáin, César González, Luisa Puig, Jorge Alcázar, Mónica Mansour y Luis Sendoya, Esther Cohen, Tatiana Bubnova. De este grupo de investigación se generan los congresos anuales sobre Poética y Semiología de 1978 a 1981 . En este espacio académico se congregan figuras como Jakobson, Teun van Dijk, André Martinet, J. M. Klinkenberg, y otros más. El órgano difusor, Acta poética, hace su aparición en 1979. Los artículos se orientan hacia la semiología literaria, la poética jakobsoniana, la glosemática. Su segunda época, bajo la dirección de César González, exhibe una tendencia hacia el dialogismo bajtiniano, la semiótica y el marxismo.

En este mismo año se funda el Centro de Ciencias del Lenguaje de la Universidad Autónoma de Puebla. Su director y fundador es Adrián Gimate-Welsh y Raúl Dorra, co-fundador. Se integran en el centro por periodos de duración diversa semiotistas como Enrique Ballón, Walter Mignolo, Per Aage Brandt, Eric Landowski, Renato Prada y José Pascual Buxó. Si bien hay un predomino de la poética -en su vertiente barthiana y jakobsoniana - también se inician estudios sobre lengua y cultura. Los primeros trabajos aparecen en la colección Signo 
y Sociedad en 1980 con el libro Lenguaje y sociedad de Adrián Gimate-Welsh. Se abordan temas del estructuralismo, pero también las relaciones entre el lenguaje, la cultura y la ideología. El análisis del discurso hace su aparición en Los dos ejes de la cruz, de Noé Jitrik. El objeto de estudio ya no es sólo el discurso literario, sino las cartas de relación de Cristóbal Colón. En 1986 se abre la revista Morphé, dirigida por Adrián Gimate-Welsh en la que se dan a conocer trabajos de A. J. Greimas, Mario Valdés, Noé Jitrik, Wlad Godzich, Wladimir Krysinski, Francois Rastier, Hermann Parret, Michel Balat, Adrián Gimate-Welsh, Raúl Dorra, Renato Prada, entre otros.

Cabe resaltar que en el Centro de Ciencias del Lenguaje se funda la primera maestría en semiótica de México. Su inicio en 1981 arranca con tres áreas de estudio y de investigación: a) lingüística, b) poética y 3) semiótica. Sus primeros egresados - titulados - de las dos primeras generaciones son ahora investigadores de la semiótica que han participado en congresos nacionales e internacionales: Raquel Gutiérrez E., Alma Yolanda Castillo, Luisa Ruiz, María Rayo Sankey García, etc. Sus temas de investigación van desde el lenguaje corporal, la cinésica y la semiótica como en el caso de Sankey García, al estudio de los lenguajes sincréticos, la semiótica plástica, la semiótica literaria, el análisis del discurso interaccional, etcétera.

En 1978, Renato Prada Oropeza funda en la Universidad Veracruzana el Seminario de Semiótica. Su orientación ha sido básicamente greimasiana, sobre todo en sus inicios. Su órgano difusor, Semiosis, ha sido la revista de semiótica más constante en su periodicidad. Sus 29 números exhiben una rica variedad de temas y orientaciones, sobre todo a partir del número 14/15 de 1985 en el que se abordan temáticas tales como la intertextualidad, teoría del género, la retórica, además de análisis en los que se aplica el método greimasiano. Sus miembros investigadores, además de su director, de origen boliviano, son: Angélica Prieto I., Sara Luz Páez Vivanco, Magda Díaz, Alfredo Pavón, Efrén Ortiz Domínguez, etc.

Los estudios semióticos hacen su aparición en la Universidad Autónoma Metropolitana, Unidad Iztapalapa, con la apertura de la revista Acciones Textuales en 1990, dirigida por Adrián GimateWelsh. En el número I aparecen temáticas relacionadas con el discurso literario, discurso terapéutico, discurso jurídico y aspectos teóricos de la semiótica estructuralista y la peirciana. En la Universidad Autónoma Metropolitana-Azcapotzalco la semiótica se instala en el ámbito del diseño gráfico. En la actualidad existe en esta universidad 
un seminario permanente sobre estudios Semióticos a cargo de Juan Manuel López.

En El Colegio de México, en el contexto del programa de doctorado en lingüística y literatura, la semiótica se hace presente mediante la impartición de cursos y conferencias a cargo de estudiosos como Thomas Sebeok y Jerzy Pelc. El apoyo a la semiótica por parte de esta institución ha sido fundamental para la realización de congresos de semiótica, al igual que la Universidad Nacional Autónoma de México y la Universidad Autónoma Metropolitana.

Pero uno de los espacios privilegiados para la semiótica lo constituyen las escuelas de comunicación 12 en todo el país. Además de la Universidad Autónoma Metropolitana, Unidad Xochimilco, tenemos las siguientes: la Universidad Iberoamericana, la Universidad Anáhuac, la Nacional Autónoma de México a través de su Facultad de Ciencias Políticas, el Instituto Tecnológico de Estudios Superiores de Occidente -además de un posgrado en Comunicación-, la Universidad de Occidente del Estado de Sinaloa y otras más.

Aunque con una vida breve, por razones de política universitaria, el Centro de Semiótica de la cultura de la Universidad Autónoma Benito Juárez de Oaxaca emerge en 1993 como un espacio previo a la creación de una Maestría en Semiótica de la Cultura, coordinado por Adrián Gimate-Welsh y José Pascual Buxó. Su actividad inicia con un diplomado en Semiótica de la Cultura y una línea de publicaciones con el nombre de Homo Signans. En este espacio se organiza el II Encuentro de Estudios de la Semiótica en noviembre de 1993.

La creación de estos centros y seminarios de investigación fue fundamental para el desarrollo de la semiótica en México, pero es necesario resaltar la existencia de otros esfuerzos en el contexto de la presencia de profesores invitados o conferenciantes en diversas instituciones. Así por ejemplo, tenemos la visita de Thomas Sebeok en El Colegio de México y en la Universidad Autónoma de Puebla; la de Jerzy Pelc, en las mismas instituciones en el año de 1991; la visita de Umberto Eco y Paolo Fabbri. De Mario Valdés, Francois Rastier, Jacques Fontanille, José Romera Castillo, Michel Arrivé, Cesare Segre, Lucia Santaella y muchos más en el contexto del VI Congreso

12 Véase mi ensayo «Semiótica y comunicación social en América Latina. El caso de México», revista Diá-logos, núm. 22, noviembre de 1988, Revista de la Federación Latinoamericana de Facultades de Comunicación Social, Lima (Perú), 1988, 6-13. 
Internacional de Estudios Semióticos que tuvo lugar en la ciudad de Guadalajara, México del 13 al 18 de julio de 1997.

\section{LOS ESTUDIOS SEMIÓTICOS}

Si quisiéramos identificar una fecha para hablar de los estudios semióticos propiamente dichos, habría que señalar la realización del I Congreso Internacional Latinoamericano que tuvo lugar en septiembre de 1985 en la Universidad Nacional Autónoma de México y la Universidad Autónoma de Puebla, organizada por la Asociación Mexicana de Semiótica, fundada en el mismo año. La temática de las ponencias ya no exhibe una hegemonía de lo literario. Ahora aparecen tópicos relacionados con la imagen, la arquitectura, los medios, la plástica, la retórica, entre otros temas. Algunos de estos trabajos fueron publicados en los números 1, 2-3 de la revista Morphé ${ }^{13}$, de la Maestría en Ciencias del Lenguaje de la Universidad Autónoma de Puebla.

Un segundo momento lo constituye el I Encuentro de Estudiosos de la Semiótica que se realiza en 1991 con el apoyo de la Universidad Nacional Autónoma de México, la Universidad Autónoma Metropolitana-Iztapalapa y El Colegio de México, bajo el impulso de la Asociación Mexicana de Semiótica. El estímulo de Jerzy Pelc fue vital en la conjunción de esfuerzos de los organizadores. La temática es ahora más amplia: la sociocrítica, los lenguajes formales, la argumentación, la comunicación, la cultura popular, la escultura, la semiótica jurídica, el discurso interaccional en la familia, discurso historiográfico, semiótica teatral, y, por supuesto, la semiótica literaria.

La segunda etapa del desarrollo de la semiótica se vio reforzada por el II Encuentro de Estudiosos de la Semiótica que se llevó a cabo en la ciudad de Oaxaca con el auspicio de la Universidad Autónoma Benito Juárez. Las conferencias magistrales nos muestran una pauta de la orientaciones que se siguieron en este coloquio: a) las teorías semióticas: iconografía y emblemática, dialogismo, semióticas especiales, poética, semiótica del infinito, la retórica, análisis del discurso;

13 «Algunas preguntas prácticas sobre el sentido» de Eric Landowski; «Semiótica y filosofía del lenguaje en la Inquiry de Bertrand Russel», de Mauricio Beuchot, etc. 
b) semiótica teatral, semiótica y ensayística, discurso femenino; c) discurso historiográfico, semiótica de los medios, discurso interaccional, semiótica de la imagen, etc. ${ }^{14}$.

Un tercer momento y decisivo de la semiótica en México fue el VI Congreso Internacional de Estudios Semióticos de la Asociación Internacional de Estudios Semióticos (IASS-AIS), que tuvo lugar en julio de 1997, organizado por la Asociación Mexicana de Estudios Semióticos (AMES) ${ }^{15}$ con el auspicio de la Universidad Nacional Autónoma de México, la Universidad Autónoma Metropolitana, El Colegio de México, la Universidad Autónoma de Puebla y la Secretaria de Cultura del Estado de Jalisco. La realización de este encuentro internacional en tierra mexicana fue sin duda un reconocimiento del desarrollo de la semiótica en México, a sus instituciones y a sus investigadores.

\section{LOS ESTUDIOS SEMIÓTICOS EN LA ACTUALIDAD}

Hablar de los estudios semióticos en México a fines del siglo XX requeriría mucho espacio, que no disponemos. Una muestra de las corrientes teóricas, los temas de estudio en relación con la semiótica, los investigadores y sus instituciones la podemos apreciar si hacemos un revisión de los trabajos de los 82 participantes del VI Congreso Internacional de Estudios Semióticos que tuvo lugar en México, en la ciudad de Guadalajara, del 13 al 18 de julio de 1997.

De acuerdo a los ejes temáticos del programa del congreso, podemos apreciar que los semiotistas mexicanos trabajaban en los siguientes líneas de investigación:

- Espacio corporal

- Espacio visual

14 Para un examen concreto de los trabajos, véase Escritos. Semiótica de la cultura, Compilador, Adrián Gimate-Welsh, México: Universidad Autónoma Benito Juárez de Oaxaca, 1994, 472 páginas.

15 Esta nueva Asociación surge de la fusión de la Asociación Mexicana de Semiología y la Asociación Mexicana de Semiótica cuyo Presidente actual es Adrián Gimate-Welsh, Presidente del VI Congreso Internacional de Estudios Semióticos donde, por cierto, fue aprobado por la Asamblea de miembros la iniciativa de que el español fuese lengua oficial de los congresos internacionales de semiótica, junto con la lengua del país sede, el francés y el inglés. 
- Espacio artístico

- Espacio fílmico

- Espacio mediático

- Espacio tecnológico

- Tiempo mítico

- Espacio musical

- Espacio simbólico

- Cosmología

- Psicosemiótica

- Sociosemiótica

- Formas de vida

- Género

- Cultura étnica

- Semiótica y lingüística

- Peirce. Modos de razonamiento

- Modelos

- Semiótica y literatura

- Análisis del discurso

- Poética

- Pragmática

- Lingüística.

Por el número de participantes, podemos apreciar que uno de los espacios privilegiados fue la antropología, pues de 82 participantes en el VI congreso Internacional de Estudios Semióticos, 10 trabajos estuvieron relacionados con la cosmología; en segundo lugar el análisis del discurso con 8 y lo relativo a las tecnologías con 7 ponencias. (véase la gráfica Ejes Semióticos. VI Congreso de la IASS-AIS al final del trabajo). Apreciamos, además, un interés por la aplicación de la semiótica y un muy reducido gusto por los aspectos teóricos.

En cuanto a las instituciones participantes ${ }^{16}$, una vez más se aprecia un claro dominio de las instituciones de la capital del país: la

16 Universidad Nacional Autónoma de México (UNAM), Universidad Autónoma Metropolitana (UAM), Benemérita Universidad Autónoma de Puebla (BUAP), Escuela Nacional de Antropología e Historia (ENAH), Colegio de México, Instituto Nacional de Antropología e Historia (INAH), Universidad Veracruzana (UV), Instituto Nacional de Bellas Artes (INBA), Instituto Tecnológico de Estudios Superiores de Monterrey (TECH), Universidad Autónoma de Nuevo León (UANL), Universidad de las Américas (UDLA), Universidad Autónoma Benito Juárez de Oaxaca (UABJO) y Universidad de Occidente (UO). 
Universidad Nacional Autónoma de México, la Universidad Autónoma Metropolitana, la Escuela Nacional de Antropología e Historia. De la universidades de provincia, la Benemérita Universidad Autónoma de Puebla tuvo la mayor presencia (véase la gráfica Presencia Institucional al final del trabajo).

De la década de los años setenta, a fines de los años noventa, el desarrollo de la semiótica ha sido notorio no sólo en términos del número de investigadores docentes que se dedican a esta disciplina en su relación a campos del conocimiento afines, sino en términos de la diversidad de enfoques. El campo de los estudios literarios sigue siendo un espacio privilegiado de la semiótica, como puede apreciarse en los títulos de las publicaciones del Seminario de Poética de la UNAM o de las revistas Semiosis, Morphé o Escritos ${ }^{17}$, pero también lo es ahora el discurso institucional: el discurso en torno a los derechos indígenas ${ }^{18}$, el discurso parlamentario ${ }^{19}$, el discurso sindical, el discurso en la familia ${ }^{20}$, el discurso en la relación médico-paciente, discurso corporal, etcétera.

Rosa Montes, por ejemplo, en sus estudios sobre los «Metaenunciados verbales y no-verbales en la organización discursiva» se apoya en los trabajos de A. Kendon y McNeill para estudiar la gestualidad según se manifiesta en la televisión mexicana; autores que también sirven de referente teórico en el estudio sobre «El galanteo. Descripción cinésica y análisis semiótico», hecho por María Rayo Sankey 21 y en «Palabra y gesto: Simbiosis en el habla infantil», de Rebeca Barriga. Alicia Poloniato en sus estudios sobre las artes visuales se funda en el trabajo de Luis Prieto. C. Metz y G. Sonesson se hacen presentes en los estudios sobre la cinematografía de Vicente Castellanos Cerda. En el estudio de la identidad nacional, JiménezOttalengo, además de tomar en cuenta las propuestas de los teóricos de la narratividad y la argumentación, $\mathbf{M}$. Foucault y $\mathbf{M}$. Weber, devienen

17 Nueva revista del Centro de Ciencias del Lenguaje de la Universidad Autónoma de Puebla.

18 Véase al respecto la tesis doctoral de Susana González Reyna sobre el discurso zapatista.

19 Ver en particular «El Presidencialismo» de Adrián S. Gimate-Welsh y Jorge Moreno Collado; «La relación iglesia-Estado» de Pedro Hernández Ornelas y María Rayo Sankey García, «La democracia» del Julieta Haidar, Luisa Béjar y Lidia Rodríguez, todos en La argumentación parlamentaria 1982-1996, México: Miguel Ángel Porrúa, 1997.

${ }_{20}$ Me refiero al tema de la tesis doctoral «Análisis de la interacción familiar» de María Rayo Sankey García; cf. asimismo su trabajo «La lógica del perdón en la discusión conyugal».

21 Revista Morphé, año 1, julio-diciembre, 1986, núm. 2, 111-137. 
fuentes de apoyo teórico para el análisis de los ensayistas Octavio Paz, Samuel Ramos y Roger Bartra. Entre las formas de vida destacan los estudios sobre el carnaval, festividades de cuaresma y otras expresiones culturales que permiten el estudio de las representaciones sobre la mujer. En este contexto se encuentran las líneas de investigación de Francisco Serrano, Alma Yolanda Castillo y A. Javier Luna Reyes quienes se apoyan las teorías del juego de L. Wittgenstein y la semiótica de las pasiones de A. J. Greimas y J. Fontanille. Estos mismos autores constituyen la base teórica del estudio del mito «Evita» que Aida Gambetta Chuck lleva a cabo en «La historia del cuerpo y el cuerpo de la historia en Santa Evita de Tomas Eloy Martínez» y del análisis de un relato de José Emilio Pacheco que realiza Roberto Flores. Bajtín, Bourdieu, Maingeneau y Kerbrat hacen acto de presencia en el estudio de Ramón Alvarado quien busca establecer una relación entre el ethos y el cuerpo (físico y social) que, como sabemos, es la influencia del ethos sobre el destinatario. La imagen del mundo, que de acuerdo con J. Lotman, se construye en textos como el ensayo -dice Blanca M. García Monsivais en «El punto de vista en el ensayo de Carlos Fuentes...»- en Fuentes es una técnica para rechazar la idea de la neutralidad en el texto artístico. Sobre el tema de las modalidades, que atañe a la lingüística pero también a la semiótica, Josefina García analiza una de las problemáticas apoyándose en la lógica epistémica de Hintikka y Partee; problemática de la subjetividad que forma parte de los estudios más remotos como los de los clásicos griegos y romanos y de la problemática del dialogismo bajtiniano.

Las referencias a los trabajos y a los autores podrían ser muchas más, pero considero que los señalamientos anteriores nos dan una imagen de la vitalidad de los estudios semióticos en México, así como de la diversidad de escuelas y tendencias teóricas. Apreciamos asimismo que no existe un claro dominio de alguna de las corrientes de pensamiento, sino que en muchos casos se articulan distintos puntos de vista para enfrentar los diferentes objetos de estudio. La realización del VI Congreso Internacional de Estudios Semióticos de la Asociación Internacional de Estudios Semióticos (IASS-AIS) ha reafirmado el proyecto semiótico en México y ha abierto nuevos horizontes que seguramente veremos en futuras reuniones de estudiosos de la semiótica. Se han roto algunas fronteras. Hemos ingresado en una nueva visión del mundo: un mundo más plural, más abierto y más sensible ante la diversidad cultural. La muestra lo constituye el ingreso del español como miembro de la familia de lenguas francas en la gran comunidad de mujeres y hombres que sólo buscan la felicidad del uno en el otro. 

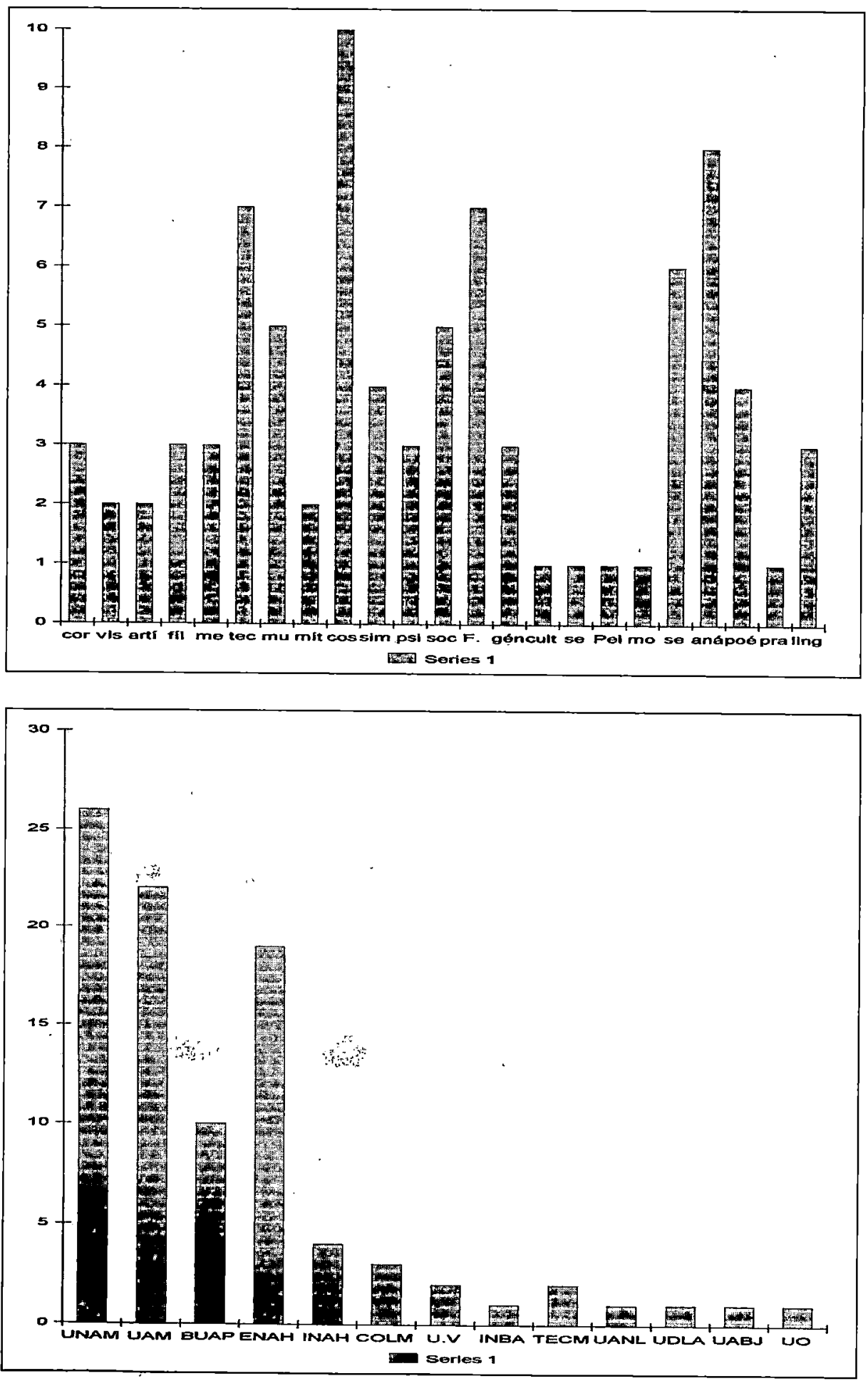


\section{Bibliografía}

AMÉzCUA, JosÉ (1987). «El espacio simbólico: El caso del teatro español del Siglo de Oro». Acta Poética 7, 36-48.

BARRIGA V., REBECA (1992a). «De las interjecciones, muletillas y repeticiones: su función en el habla infantil». En Reflexiones Lingüisticas y Literarias. México: El Colegio de México, 99-114.

- (1992b). «Significados y sentidos en el habla infantil». Varia Lingüistica y Literaria, 327-342.

Barriga V., Rebeca y Butragueño, Pedro M. (editores) (1997). Varia Lingǘstica y Literaria. México: El Colegio de México.

BERISTÁIN, HELENA (1982a). Análisis estructural del relato literario. México: UNAM.

- (1982b). «Las unidades distribucionales en el análisis del relato». Acta Poética 4-5, 235-252.

Beuchot, Mauricio (1980a). «Análisis semiótico de la metáfora». Acta Poética 2, 113-25.

- (1980b). «La doctrina tomista sobre el signo: Domingo de Soto, Francisco de Araujo y Juan de Santo Tomás». Crítica 12, 39-60.

- (1986). «Semiótica y filosofía del lenguaje en la Inquiry de Bertrand Russell». Morphé 2, 73-87.

- (1987a). Aspectos históricos de la semiótica y la filosofía del lengua$j e$. Cuadernos del Seminario de Poética 11. México: UNAM.

- (1987b) «Hermenéutica y retórica en Hans Georg Gadamer». Semiosis $18,141-48$.

- (1991). La filosofía del lenguaje en la edad media. Cuaderno $38 \mathrm{del}$ Seminario de Poética. México: UNAM.

Bubnova, TATIANA (1982). «El texto literario, producto de la interacción verbal. Teoría del enunciado de M. Bajtín». Acta Poética 4/5, 215-33.

- (1987). «En busca de una poética del mito». Acta Poética 7, 95-122.

Carbó, Teresa (1984). «El debate indigenista en México. Un ejemplo de análisis de discurso parlamentario». Discurso 1, 55-82.

- (1993a). «Determinaciones discursivas sobre episodios interaccionales en situación de debate». Morphé 8, 27-52.

- (1993b). «Nosotros que nos quisimos tanto... La escena discursiva en la Cámara de Diputados». Discurso. Teoría y análisis 14, 69-96.

- (1997). «Lázaro Cárdenas: coyuntura, persona, pronombre». En Varia Lingüística y Literaria (editores Rebeca Barriga y Pedro Martín Butragueño). México: El Colegio de México, 225-242.

CASTAÑOS, FERNANDO (1992). «Ilocución, discurso». Teoría y análisis 13, 25-34.

Castillo, Alma Yolanda, Huerta C., Elisa, Huerta C. Juan (1995-6). «Entre semiótica y antropología: el rito de la ofrenda a los volcanes». Morphé 13-14, 319-354. 
Castillo alma, Yolanda; Dávila, Joel y Serrano, Francisco (1988). «Hacia una topología del carnaval de Huejotzingo». Escritos 3-4, 29-47

DORRA, RAúl (1983). «El tema del sujeto Morirás lejos». Semiosis 11, 111-43.

- (1987). «Los deleites abstractos y el placer multiforme». Morphé 3-4, Universidad Autónoma de Puebla, 149-158.

- (1991). «Que la semiótica puede ser también una dicha». Discurso. Teoría y análisis $11,75-89$.

- (1993a). «Los relatos literarios: entre la proliferación y la clasificación». Acta Poètica 11, 35-48.

- (1993b). «¿La retórica contra la Magdalena?».Discurso. Teoría y análisis 15, 59-84.

FIDELHOLTZ, JAMES (1987). «El sistema de acentuación gráfica de la lengua española». Morphé 3-4, 185-199.

FLORES, ROBERTO (1992). «La alteración - los juegos de la reedición en la interacción—». Semiosis 26-29, 319-336, Universidad Veracruzana, México.

Gambetta Ch., AidA (1993). «Entre el espejo y la máscara: H. Bustos Domecq». Escritos 8, 99-110.

GARCía FAJARDO, JOSEFINA (1985). El sentido de los sintagmas nominales y los tipos de predicación. México: Instituto Nacional de Antropología e Historia, Secretaría de Educación Pública.

- (1992). «Las variaciones de sentido, los sujetos y el universo del discurso». En Reflexiones lingüisticas y literarias, El Colegio de México, 231-250.

Garza Cuarón, Beatriz (1978). La connotación: Problemas de significado. México: El Colegio de México.

- (1980). «Fijeza y variabilidad del significado». Acta Poética 2, 59-72.

- (1981). «La ambigüedad: Tipos y aspectos». Diálogos 100, 17-72.

- (1985). «La referencialidad como concepto lingüístico». Nueva Revista de Filología Hispánica 34, 1-22.

- (1992). «Las lenguas y las aportaciones de la lingüística en Hispanoamérica». Semiosis 26-29, 219-236.

- (1995). «El olvido del contexto en el análisis del significado». Cruzeiro Semiotico 22/25, 173-180.

Gimate-Welsh, AdRiÁN (1980). Lenguaje y sociedad, signo y Sociedad 1. Universidad Autónoma de Puebla.

- (1984). «Análisis del proceso religioso a través del análisis del discurso». Dialéctica 16, 83-97, Universidad Autónoma de Puebla,

- (1986). «Semiótica y análisis del discurso». Morphé 1, 145-144.

- (1987). «Las investigaciones semióticas en México». Morphé 3-4, 135-148.

- (1988). «Semiótica y comunicación social en América Latina. El caso de México».Diá-logos (Perú), 6-13.

- (1990a). Introducción a la lingüística. Modelos y reflexiones actuales. México: Fondo de Cultura Económica. 
- (1990b). «Pugna por la democracia. El discurso priísta y neocardenista». Acciones Textuales (Universidad Autónoma Metropolitana), Iztapalapa, 1, 97-112.

- (1990c). «Elementos fundantes de la semítica greimasiana». Signos. Universidad Autónoma Metropolitana-Iztapalapa, tomo 1, 265-279.

- (1992). «Democracia. Entidad de dos caras». Semiosis 26-29, 249 266. Universidad Veracruzana.

- (1993a). «El lugar de A. J. Greimas en la construcción de la semiótica». Acciones Textuales 4-5, v-xvi.

- (1993b). Itinerarios. Del campo a la teoría. Oaxaca: Universidad Autónoma Benito Juárez de Oaxaca.

- (1994a). Introducción a la lingüística. México: Fondo de Cultura Económica.

- (1994b). Escritos. Semiótica de la cultura, comp. Universidad Autónoma Benito Juárez de Oaxaca.

- (1995a). Democracia. Entidad de dos caras. El discurso del PRI y la corriente democrática. Coyoacán, BUAP, UABJO.

- (1995b). «A Semiotic Reading of Octavio Paz'Essays». Cruzeiro Semiotico (Porto), 22-25, 399-410.

- (1997a). «Correspondencias y símbolos en el arte de Rufino Tamayo». Varia Lingüística y Literaria. El Colegio de México, 287-306.

- (1997b). La argumentación parlamentaria, Coordinador. Miguel Ángel Porrúa, Serie IV tomo II.

- (1998). Los paradigmas de la semiótica. Serie Semiótica, Universidad Autónoma Metropolitana-Azcapotzalco.

Gimate-Welsh, A. y MARROQú́N, ENRIQue (1985). Lenguaje, ideología y clases sociales. Las vecindades de Puebla. México: Universidad Autónoma de Puebla.

GiméNez, Gilberto (1983). Discurso, poder y estado. México: Universidad Nacional Autónoma de México.

- (1993). «Análisis argumentativo y sociocrítica de la ficción: Dos aproximaciones discurso». En Pervivencia del signo. México: UNAM, UAM-I, El Colegio de México.

GonZÁLEZ, CÉsAR (1979). «La semántica de Roman Jakobson». Acta Poética

$1,71-90$.

- (1980). «De la semiología al análisis del discurso». Acta Poética 2,73-111.

- (1981). «Algunas consideraciones sobre la expresión Discurso literario». Acta Poética 3, 163-79.

- (1982). «Función de la teoría en los estudios literarios». Cuadernos del Seminario de Poética 7, UNAM.

- (1986). Imagen y sentido. Elementos para una semiótica de los mensajes visuales. Cuadernos del Seminario de Poética, UNAM.

- (1988). «Notas sobre concepto de valor». Semiosis 20, 207-226.

- (1992). «Concepción medieval del tiempo». Discurso. Teoría y análisis 13, 85-106. 
GUTIÉRREZ, RAQUEL (1986). «La focalización. Génesis y desarrollo de un concepto». Semiosis 17, 113-35.

- (1990). «Acerca de la crónica». Semiosis 24, 239-254.

- (1992). «Espacio y sujeto en la tercera Carta de Relación». Semiosis 26-29, 377-396.

- (1994). «Fundamentos para una teoría del discurso literario femenino». En Escritos. Semiótica de la cultura, Universidad Autónoma Benito Juárez de Oaxaca, 185-208.

Jiménez-OtTAlengo (1986). «Semiotics in México». En The Semiotic Web, Thomas Sebeok and Jean-Umiker Sebeok (eds.) 359-67. New York, London: Plenum Press.

JiMÉNEZ-OtTAlENGO, REgina (comp.) (1984a). «El enfoque pragmático de las ideologías y sus antecedentes». En Sociología y semiología, 47-60. México: UNAM.

- (1984b). «El signo desde el punto de vista sociológico». En Sociología y semiología, 41-46. México: UNAM.

- (1985). «Configuraciones valorativas de la interacción de los extranjeros en México». Semiología 2, 84-119.

- (1986). «Aproximaciones sociosemiológicas de la incorporación de los mazahuas a la vida urbana». Semiótica 3, 69-82.

JiMÉNEZ-OTTALENGo, REGiNA y RoDRíGUEZ, M.L. (1980). «Reflexiones acerca de la semiología, el problema del signo y la realidad». En Lecturas de semiología 9, 73-89. México: UNAM.

JITRIK, NoÉ (1986). «Discursividad, discurso, análisis». Morphé 1, 43-51.

- (1997). «La escritura en el hueco del deseo. La semiosis, la falta y la teoría del signo». En Varia lingüística y literaria. México: El Colegio de México.

MARCOS ORTEGA, JOSÉ (1986). «Figuratividad y figurativización en los anuncios publicitarios». Morphé 2, 11-15.

- (1992). «Evidencia neurofisiológica de los procesos de categorización léxica y acceso al significado». En Reflexiones lingüístico literarias, 187-211, vol. 1. México: El Colegio de México.

- (1997). «Aspectos neurofisiológicos de los procesos de acceso al léxico. Efectos de la lexicalidad y la frecuencia». En Varia lingüística y literaria, 383-410. México: El Colegio de México.

Mier, RAYMundo (1993). «Derroteros del análisis». Discurso. Teoría y análisis 14, 31-68.

MonTES MiRo, Rosa G. (1986). «Factores discursivos en el análisis de los pronombres personales sujeto en español». Morphé 2, 45-71.

- (1987). «Secuencias de clarificación en conversaciones con niños». Morphé 2-4, 167-184.

- (1994). «El desarrollo de conocimientos metalingüísticos en el niño». Escritos. Semiótica de la cultura, 277-99. Oaxaca: Universidad Autónoma Benito Juárez de Oaxaca. 
- (1996). «Relaciones entre expresiones verbales y no verbales en la organización del discurso». Estudios de Lingüística Aplicada 19-20, 253-272, UNAM.

- (1997a). «Adquisición de estrategias de reparación». Signo y Seña, Argentina.

- (1997b). «Explicar: el verbo en discurso referido». Estudios de Lingüística Aplicada 23-24, 255-263.

Quesada, RAÚl (1984). «De la lógica y la lingüística al discurso». Discurso.

Teoría y análisis 3, 9-30.

- (1993). «Leer». Discurso. Teoría y análisis, 14, 1-20.

PASCUAL BUXó, JosÉ (1976). «Premisas a una semiología del texto literario». Anuario de Letras 14, 175-200. México: UNAM.

- (1978). Introducción a la poética de Roman Jakobson. Cuadernos del Seminario de Poética 1. México: UNAM.

- (1979). «Estructuras lingüísticas y paradigmas ideológicos». Acta Poética 1, 2-22.

- (1980). «Sincretismo, homología, ambigüedad referencial». Acta Poética 2, 41-57.

- (1981a). «La estructura del texto semiológico». Acta Poética 3, 37-55.

- (1981b). «La semantización discursiva de los componentes mínimos del signo». Semiosis 6, 27-38.

- (1982). «Las articulaciones semánticas del texto literario». Acta Poética 4-5, 53-76. México: UNAM.

- (1984a). Las figuraciones del sentido. México: Fondo de Cultura Económica.

- (1984b). «Texto y discurso: Semiótica e ideología». Discurso 1, 45-62.

- (1990). «La estructuración semiótica de las ideologías». Acciones Textuales 1, 37-54.

- (1993a). «Bernardo de Balbuena. El arte como artificio». Acciones Textuales 4-5, 205226.

- (1993b). El enamorado de sor Juana. México: Estudios de Cultura Literaria novohispana 2, UNAM.

- (1994). «Iconografía y emblemática». En Escritos. Semiótica de la cultura, Universidad Autónoma Benito Juárez de Oaxaca, 21-42.

Pimentel Luz Aurora (1991). «Sobre la lectura». Acta Poética 11, 49-80.

PRAda O., Renato (1977). «El cine, un lenguaje». La palabra y el hombre

21, Universidad Veracruzana, 56-60.

- (1978). «El estatuto del personaje». Semiosis 1, 25-45

- (1983). «Co-texto y con-texto en El Apando de José Revueltas». Semiosis 10, 147-165.

- (1985). «Los elementos pragmáticos del nivel discursivo: el narrador y el narratario». Semiosis 14-15, 3-35

- (1986). «El estatuto semiótico del texto narrativo». Morphé 1, 81-102.

- (1987). «El personaje: de la discursivización a la textualización». Morphé 3-4, 119-134. 
- (1988). «El nivel del contenido del discurso narrativo-literario». Semiosis 20, 3-72.

- (1990). «La teoría del distanciamiento y el discurso narrativo-literario». Acciones Textuales 2, 155-174.

- (1992). «Configuración de "el otro" en el discurso testimonio». Semiosis 26-29, 423-434.

- (1994). «Literatura y sociedad». Escritos. Semiótica de la Cultura, 73108.

Rivera, Ligia (1994). «Tenue dominio de los dioses cristianos. Análisis semiótico de un macrorrelato indio». Escritos 10, 101-138.

RODRf́GUEZ A., LIDIA (1982). «Perspectivas en la pragmática actual». Discurso. Teoría y análisis 11, 62-74.

RUZz M., LuISA (1986). «El discurso mariano: Espacio sincrético en Latinoamérica». Morphé 2, 21-29.

SANKEY GaRCIA, MARÍa RAYO (1986). «El galanteo: Descripción cinésica y análisis semiótico». Morphé 2, 111-37.

- (1990). «Cinésica y semiótica». Acciones Textuales 1, 183-184.

- (1990). «La negociación en el discurso terapéutico». Acciones Textuales 2, 69-88.

- (1992). «Aspectos no verbales de la comunicación». Semiosis 26-29, 283-294.

- (1993). «Microanálisis de los procesos comunicativos: La interacción familiar como discurso». Pervivencia del signo, UNAM, UAM, Colmex, 89-92.

- (1994). «Las formas argumentativas en la resolución de conflictos: Análisis de un caso». Escritos. Semiótica de la cultura, UABJO, 299306.

- (1997). «La dimensión dialógica del debate parlamentario». En La Argumentación (Coordinador Adrián Gimate-Welsh). México: Miguel Ángel Porrúa.

- (1998). El discurso silencioso. Oaxaca: Universidad Autónoma de Puebla-Universidad Autónoma Benito Juárez de Oaxaca.

SERRANO O. FRANCISCO (1988). «El recorrido narrativo: eje fundamental de las estructuras narrativas». Escritos 3-4, 3-10, Centro de Ciencias del Lenguaje, Universidad Autónoma de Puebla.

ZaVAla, LAURo (1991). «Entre la seducción y la historia: notas sobre la recepción del cine y la pintura». Acta Poética 11, 81-96.

- (1992). «Para nombrar las formas de la ironía». Discurso. Teoría y análisis 13, 59-84. 